\title{
Intelligent Motion Compensation for Improving the Tracking Performance of Shipborne Phased Array Radar
}

\author{
J. Mar, ${ }^{1,2}$ K. C. Tsai, ${ }^{2}$ Y. T. Wang, ${ }^{2}$ and M. B. Basnet ${ }^{2}$ \\ ${ }^{1}$ Communication Research Center, Yuan-Ze University, 135 Yuan-Tung Road, Jungli, Taoyuan 320, Taiwan \\ ${ }^{2}$ Department of Communications Engineering, Yuan-Ze University, 135 Yuan-Tung Road, Jungli, Taoyuan 320, Taiwan
}

Correspondence should be addressed to J. Mar; eejmar@saturn.yzu.edu.tw

Received 26 March 2013; Accepted 22 May 2013

Academic Editor: Ulrich Nickel

Copyright (c) 2013 J. Mar et al. This is an open access article distributed under the Creative Commons Attribution License, which permits unrestricted use, distribution, and reproduction in any medium, provided the original work is properly cited.

\begin{abstract}
The shipborne phased array radar must be able to compensate the ship's motion and track the maneuvering targets automatically. In this paper, the real-time beam pointing error compensation mechanism of a planar array antenna for ship's motion is designed to combine with the Kalman filtering. The effect of beam pointing error on the tracking performance of shipborne phased array radar is examined. A compensation mechanism, which can automatically correct the beam pointing error of the planar antenna array, is proposed for shipborne phased array radar in order to achieve the required tracking accuracy over the long dwell time. The automatic beam pointing error compensation mechanism employs the parallel fuzzy basis function network (FBFN) architecture to estimate the beam pointing error caused by roll and pitch of the ship. In the simulation, the models of roll and pitch are used to evaluate the performance of beam pointing error estimation mechanism based on the proposed parallel FBFN architecture. In addition, the effect of automatic beam pointing error compensation mechanism on the tracking performance of adaptive extended Kalman filter (AEKF) implemented in ship borne phased array radar is also investigated. Simulations find out that the proposed algorithms are stable and accurate.
\end{abstract}

\section{Introduction}

Beam pointing error caused by ship motion over the ocean affects the tracking performance of the shipborne phased array radar. The antenna stabilization to achieve the beam pointing accuracy over the long dwell time is an important issue for shipborne phased array radar [1]. There are two ship motion compensations: compensation for rotational motion (i.e., pitch, roll, and heading angle) and compensation for translational motion (i.e., radial speed relative to the earth). Figure 1 shows the shipborne phased array radar system [2,3], which consists of the gyro sensor, beam steering controller (BSC), array antenna, radar control computer (RCC), digital signal processor (DSP), transmitter and receiver (XMTR/ RCVR), and display. Gyro sensor provides pitch, roll, heading angles of the ship, speed, course, vertical velocity of antenna installed on the ship at a data rate of $100 \mathrm{~Hz}$. To compensate the translational motion, the speed, course, and vertical velocity acquired from the Gyro sensor are averaged for the duration of radar-dwell time. Then the Doppler shift introduced by the translational motion is calculated to compensate the radial velocity estimated in the digital signal processor (DSP). The radar control computer (RCC) provides the target locations relative to earth, scheduled beam directions, and predicted beam pointing error to the beam steering controller (BSC), which compensate the beam pointing error and controls the phased array antenna to point the beam at the target direction relative to the ship coordinates.

The idea of the extended Kalman filter (EKF) was originally proposed by Stanley Schmidt so that the Kalman filter could be applied to nonlinear spacecraft navigation problems [4]. The well-known conventional EKFs give the optimal solution but require synchronous measurements, an accurate system model, and exact stochastic noise characteristics. Thus, the performance of EKF with incomplete information and asynchronous sensors measurements may be degraded or even diverged. In order to reduce the effect of noise variance uncertainty, adaptive extended Kalman filter (AEKF) is proposed to overcome this drawback. 


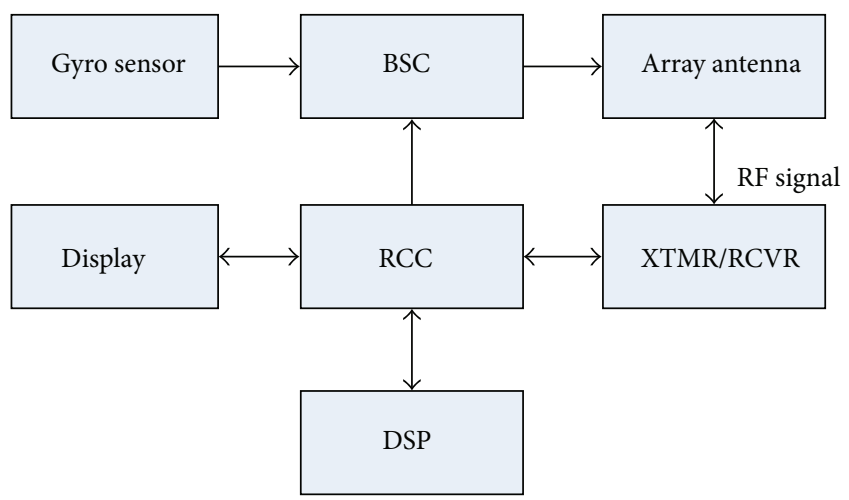

FIGURE 1: High level structure of shipborne phased array radar.

In this paper, we focus on the design of an automatic beam pointing error correction mechanism based on the proposed parallel fuzzy basis function network (FBFN) architecture, which predicts the beam pointing error caused by roll and pitch in real time. The AEKF algorithm is used to track the air target in three-dimensional space, and the covariance matrix values of process noise and measurement noise are adaptively estimated to ensure the AEKF convergence.

The rest of this paper is organized as follows: the model of ship rotational motion, coordinates transform and planar array antenna of shipborne phased array radar are described in detail in Section 2. The architecture of parallel FBFN beam pointing error estimator is presented in Section 3. The AEKF algorithm is described in Section 4. In Section 5, the tracking performance of shipborne phased array radar using the proposed beam pointing error compensation method is simulated for six different cases. Finally, we conclude the paper in Section 6.

\section{Ship Rotational Motion Compensation}

The shipborne phased array radar must be able to compensate the ship's motion and track the maneuvering targets automatically. The algorithm of real-time compensating for ship's motion is designed to combine with the Kalman filtering. The block diagram of rotational motion compensation system for shipborne phased array radar is shown in Figure 2 [3], which consists of beam pointing error prediction, ship coordinates/earth coordinates conversion, earth coordinates/ship coordinates conversion, and adaptive extended Kalman filter $(\mathrm{AEKF})$. Assume that the beam steering angle is $\left(\theta_{0}, \phi_{0}\right)$, the antenna point angle offset caused by the ship motion (roll, pitch) is $\left(\theta_{e}, \phi_{e}\right)$, and then the current antenna pointing angle is $\left(\theta_{E}, \phi_{E}\right)$ as

$$
\begin{gathered}
\theta_{E}=\theta_{0}+\theta_{e}, \\
\phi_{E}=\phi_{0}+\phi_{e} .
\end{gathered}
$$

If the beam pointing error is predicted as $\left(\Delta \theta_{E}, \Delta \phi_{E}\right)$, then the beam pointing angle is corrected as

$$
\begin{aligned}
& \theta_{E C}=\theta_{0}+\theta_{e}-\Delta \theta_{E}, \\
& \phi_{E C}=\phi_{0}+\phi_{e}-\Delta \phi_{E} .
\end{aligned}
$$

The $\left(\theta_{E C}, \phi_{E C}\right)$ is approximated to $\left(\theta_{0}, \phi_{0}\right)$.
TABLE 1: Sea state parameters.

\begin{tabular}{lcccc}
\hline Sea state & \multicolumn{2}{c}{ Roll } & \multicolumn{2}{c}{ Pitch } \\
& $A_{R}\left(^{\circ}\right)$ & $T_{R}(\mathrm{sec})$ & $A_{P}\left(^{\circ}\right)$ & $T_{P}(\mathrm{sec})$ \\
\hline 4 & 2 & 8 & 0.4 & 5 \\
5 & 10 & 10 & 2 & 7 \\
6 & 18 & 13 & 4 & 8 \\
\hline
\end{tabular}

2.1. Ship Rotational Motion Model. Ship is affected by the waves in the ocean, resulting in six degrees of freedom of movement. In this paper, assuming zero yaw angle, the simplified roll and pitch model $[1,5]$ are adopted to describe the ship rotational motion in the earth coordinates. Ship's rotational motion is modeled with sinusoidal signal. The roll angle is

$$
\phi_{R}(t)=A_{R} \sin \left(\omega_{R} t\right)+n_{R} .
$$

The pitch angle is

$$
\theta_{P}(t)=A_{P} \sin \left(\omega_{P} t\right)+n_{P},
$$

where $A_{R}$ and $A_{P}$ are the amplitude of ship's roll and pitch angles, $n_{r}$ and $n_{P}$ are assumed to be zero mean Gaussian noise, $T_{R}$ and $T_{P}$ are the roll and pitch periods, and $\omega_{R}=2 \pi / T_{R}$ and $\omega_{P}=2 \pi / T_{P}$ are the roll and pitch angular frequencies. The beam pointing errors caused by other unknown factors, including ship's traveling direction changing and whether changing, in the actual ship navigation environment will be considered in the standard deviation of the noise terms. The amplitude and period parameters of sea states 4, 5, and 6 are listed in Table 1 [1].

2.2. Coordinates Transform. Since the phased array antenna is installed on the ship, the beam steering control employs the ship body coordinates. But the target tracking of Kalman filter employs the Earth coordinates. The Euler coordinates transform formula is used to convert antenna beam pointing angle relative to the earth $\left(\theta_{E}, \phi_{E}\right)$ into antenna beam pointing angle relative to the ship body $\left(\theta_{A}, \phi_{A}\right)[5]$ as

$$
\left[\begin{array}{c}
\sin \theta_{A} \cos \phi_{A} \\
\sin \theta_{A} \sin \phi_{A} \\
\cos \theta_{A}
\end{array}\right]=T(\phi) T(\theta) T(\psi)\left[\begin{array}{c}
\sin \theta_{E} \cos \phi_{E} \\
-\sin \theta_{E} \sin \phi_{E} \\
\cos \theta_{E}
\end{array}\right],
$$

where the coordinates transform matrices are defined as

$$
\begin{gathered}
T(\phi)=\left[\begin{array}{ccc}
1 & 0 & 0 \\
0 & \cos \phi & \sin \phi \\
0 & -\sin \phi & \cos \phi
\end{array}\right] \\
T(\theta)=\left[\begin{array}{ccc}
\cos \theta & 0 & \sin \theta \\
0 & 1 & 0 \\
-\sin \theta & 0 & \cos \theta
\end{array}\right] \\
T(\psi)=\left[\begin{array}{ccc}
\cos \psi & -\sin \psi & 0 \\
\sin \psi & \cos \psi & 0 \\
0 & 0 & 1
\end{array}\right],
\end{gathered}
$$

where $\phi$ is the ship roll angle, $\theta$ is the ship pitch angle, and $\psi$ is the ship yaw angle. 


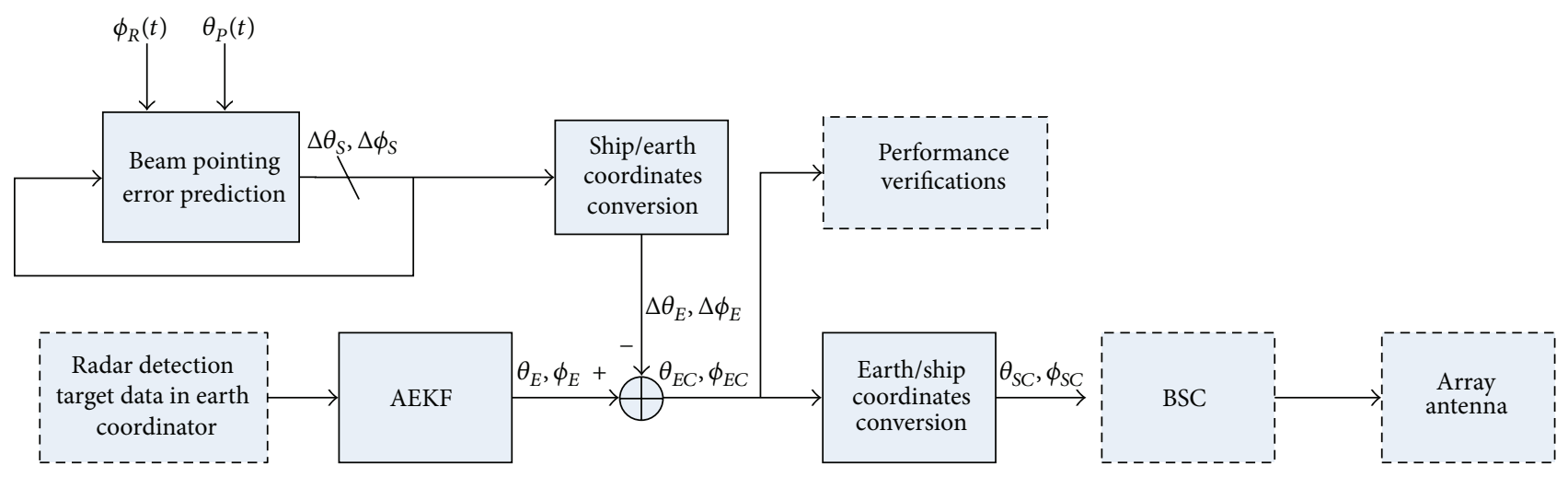

FIGURE 2: System diagram of rotational motion compensation for AEKF of shipborne phased array radar.

2.3. Planar Array Antenna. An $M \times N$ element planar array, as shown in Figure 3, is designed for the shipborne phased array radar system, which includes the beamforming (BF) mode and direction of arrival (DOA) mode. The planar array can produce multibeams in the azimuth and elevation by using the beamformer network (BFN), which consists of a set of power dividers and phase shifters. The planar array using amplitude comparison method [6] generates the difference signal patterns for the DOA estimation. The difference signals obtained from two neighboring beams can measure the DOA of the subscriber signal of the shipborne phased array radar system. The beam pattern is expressed as $[6,7]$

$$
\begin{aligned}
A F(\theta, \phi)= & \sum_{n=1}^{N} I_{1 n}\left[\sum_{m=1}^{M} I_{m 1} e^{j(m-1)\left(k d_{x} \sin \theta \cos \phi+\beta_{x}\right)}\right] \\
& \quad \times e^{j(n-1)\left(k d_{y} \sin \theta \sin \phi+\beta_{y}\right)} \\
= & S_{x m} S_{y n},
\end{aligned}
$$

where

$$
\begin{gathered}
S_{x m}=\sum_{m=1}^{M} I_{m 1} e^{j(m-1)\left(k d_{x} \sin \theta \cos \phi+\beta_{x}\right)} \\
S_{y m}=\sum_{n=1}^{N} I_{1 n} e^{j(n-1)\left(k d_{y} \sin \theta \sin \phi+\beta_{y}\right)} \\
\beta_{x}=-k d_{x} \sin \theta_{t} \cos \phi_{t} \\
\beta_{y}=-k d_{y} \sin \theta_{t} \sin \phi_{t}
\end{gathered}
$$

where $I_{m 1}=I_{1 n}=$ Chebyshev weighting [5], $M=$ the number of array elements in the $x$-axis, $N=$ the number of array elements in the $y$-axis, $\phi_{t}=$ the azimuth steering angle of the main beam, $\theta_{t}=$ the elevation steering angle of the main beam, $d_{x}=$ the interelement spacing in the $x$-axis, and $d_{y}=$ the interelement spacing in the $y$-axis.

The phase of the RF signal at each array element is adjusted to steer the beam to the coordinates $\left(\theta_{t}, \phi_{t}\right)$. For $M=N=$ 6 and $d x=d y=\lambda / 2$, the computed antenna radiation pattern of a beam steered to $(\theta, \phi)=\left(0^{\circ}, 0^{\circ}\right)$ is shown in Figure 4 . The side-lobe suppression is $-30 \mathrm{~dB}$ by using the

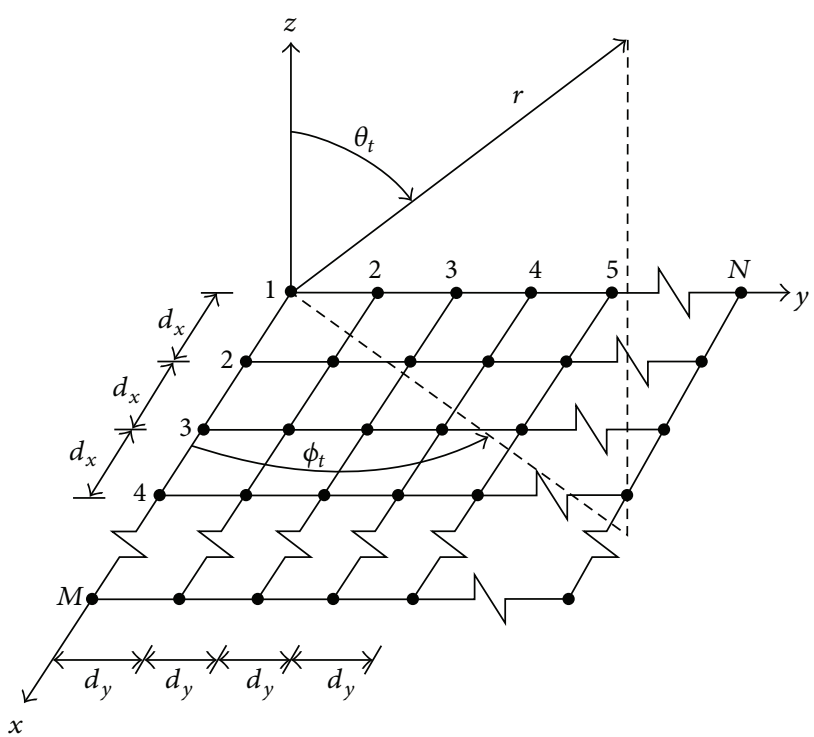

Figure 3: $M \times N$ planar array antenna.

Chebyshev weighting $=\left[\begin{array}{llllll}0.54 & 0.78 & 1 & 1 & 0.78 & 0.54\end{array}\right]$. The $6 \times$ 6 planar array antenna is chosen to simulate the beam pointing error compensation and the tracking accuracy of maneuvering target position $\left(\theta_{t}, \phi_{t}\right)$.

\section{Parallel FBFN Architecture}

The beam pointing error of the shipborne phased array radar over the ocean is always changing from time to time, so it is necessary to intelligently predict and compensate the beam pointing error through the collected ship's roll and pitch angles data. For reducing the computation load and meeting the real-time requirement of the automatic beam pointing error correction system, a simplified parallel FBFN architecture, which makes use of the roll angle and pitch angle measurements mapped onto the beam pointing error model to nonlinearly estimate the angle error of the planar antenna array, is proposed. The parallel FBFN architecture is shown in Figure 5, which is implemented with four-layer feed forward network. The input vectors $\mathbf{A}_{n}$ including $K$ beam-pointing 


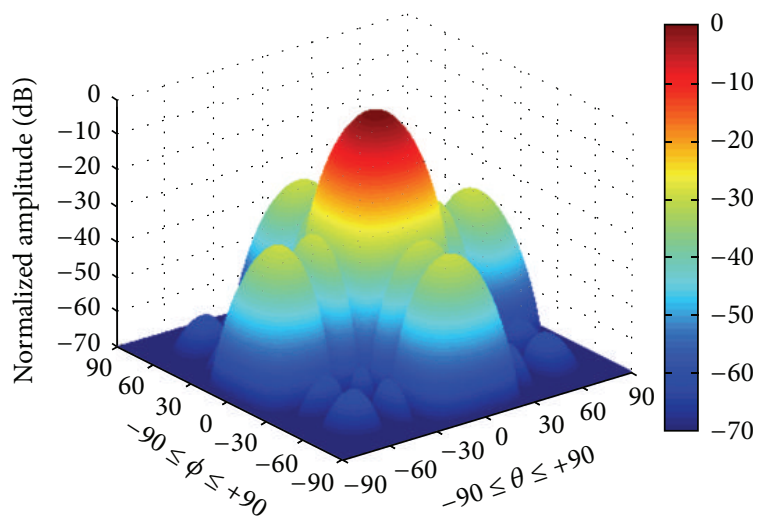

FIgURE 4: Three-dimensional antenna radiation pattern for $6 \times 6$ planar array antenna.

angles at the time $n, n-1, \ldots, n-K$ are input to membership functions to determine its membership grade.

The RLS algorithm and the FBFN algorithm with the properly normalized Gaussian membership function are employed to automatically correct the beam pointing error caused by the ship's roll angle and pitch angle. The beam pointing error correction mechanism using the RLS algorithm is realized with a $K$ th order adaptive prediction filter. The RLS algorithm is summarized as follows [8].

Initialize $\mathbf{P}(0)=\delta_{R}{ }^{-1} \mathbf{I}, \widehat{\mathbf{w}}(0)=\mathbf{0}$.

At each instant of time, $n=1,2, \ldots$, compute $M \times M$ inverse correlation matrix as

$$
\mathbf{P}(n)=\xi^{-1} \mathbf{P}(n-1)-\xi^{-1} \mathbf{k}(n) \theta^{H}(n) \mathbf{P}(n-1) .
$$

The $M \times 1$ gain vector is

$$
\mathbf{k}(n)=\frac{\xi^{-1} \mathbf{P}(n-1) \theta(n)}{1+\xi^{-1} \theta^{H}(n) \mathbf{P}(n-1) \theta(n)} .
$$

The predicted correction angle is obtained by

$$
Y(n)=\theta^{H}(n) \widehat{\mathbf{w}}(n-1) .
$$

The estimation error is

$$
f_{M}(n)=\theta(n+1)-Y(n),
$$

where $\theta_{P}(n+1)$ is the beam point error at $n+1$ time instant. The update weightings of the prediction filter are

$$
\widehat{\mathbf{w}}(n)=\widehat{\mathbf{w}}(n-1)+\mathbf{k}(n) f_{M}(n) .
$$

The RLS algorithm is convergent if the following condition is satisfied:

$$
\delta_{r}<0.01 \sigma_{\theta}^{2},
$$

where $\sigma_{\theta}^{2}$ is the variance of the Gaussian distribution of beam pointing error for pitch angle. The forgetting factor $\xi$ is a constant in $(0,1)$. Here we assume that $\delta_{r}=0.0008, \xi=1$, and $M=K$.

The FBFN-based beam pointing error correction mechanism of the LMDS system is shown in Figure 7. The FBFN is a 4-layer feed forward network. The input vectors $\vec{A}$ include $K$ beam-pointing angles at $n, n-1, \ldots, n-K$. In the first layer, the rule base $\mu_{i}(\vec{x})$ contains Takagi and Sugeno's type fuzzy if-then rules $[9,10]$. The normalized Gaussian-shaped membership functions are

$$
\mu_{i}(\vec{x})=\exp \left\{-\frac{\left\|\vec{x}-\vec{c}_{i}\right\|^{2}}{\sigma_{i}^{2}}\right\}, \quad i=1,2, \ldots, m,
$$

where the input vector $\vec{x}$ could be pitch $\vec{\theta}_{p}$ or roll $\vec{\theta}_{r}$; " $\|$ " represents Euclidean form. $\vec{c}_{i}, i=1,2, \ldots, m$ are the mean vectors of the Gaussian membership functions for ship's roll or pitch, which are generated by different sea states as

$$
\vec{c}_{i}=A \sin \left(\omega n_{i}\right), \quad i=1,2,3, \ldots, m,
$$

where the discrete sampling time is $n_{i}=i \Delta T=i / f_{s}$ and $f_{s}$ is the sampling frequency. Each mean vector consists of $K$ samples of the normalized Gaussian function with corresponding mean to the sampled sinusoidal signal value. The standard deviation $\sigma_{i}$ determines the range of Gaussian membership functions for ship's roll or pitch.

The input pitch vector is given by

$$
\vec{x}=\vec{\theta}_{p, n}=\left[\theta_{p}(n), \theta_{p}(n-1), \ldots, \theta_{p}(n-K)\right]^{T},
$$

where " $T$ " means transpose. The ratio of the $i$ th rule's firing strength to the sum of all rule's firing strengths is calculated in the $i$ th node as

$$
\bar{\mu}_{i}=\frac{\mu_{i}}{\sum_{i} \mu_{i}}, \quad i=1, \ldots, m .
$$

For convenience, the outputs of this layer are called "normalized firing strength." The output of the third layer is

$$
O_{i, n}^{3}=\vec{\mu}_{i, n} \times f_{i, n} .
$$

The consequent parameters are

$$
f_{i}=\sum_{k=0}^{K} \alpha_{i, k} \theta_{p}(n-k)+\alpha_{i, K+1}, \quad i=1,2, \ldots, m,
$$

where $\alpha_{i, k}$ is the coefficients of the consequent parameters. The consequent parameter vector is

$$
\vec{\alpha}=\left[\alpha_{i, k} \mid i=1,2, \ldots, m ; k=0,1, \ldots, K\right]_{(m(K+2)) \times 1}^{T} .
$$

The output of the fourth layer is obtained as

$$
Y(n)=\frac{\sum_{i=1}^{m} \vec{\mu}_{i, n} f_{i, n}}{\sum_{i=1}^{m} \vec{\mu}_{i, n}} .
$$

The matrix form of FBFN beam pointing error estimator can be expressed as

$$
\mathbf{W} \vec{\alpha}=\vec{Y}
$$

where 


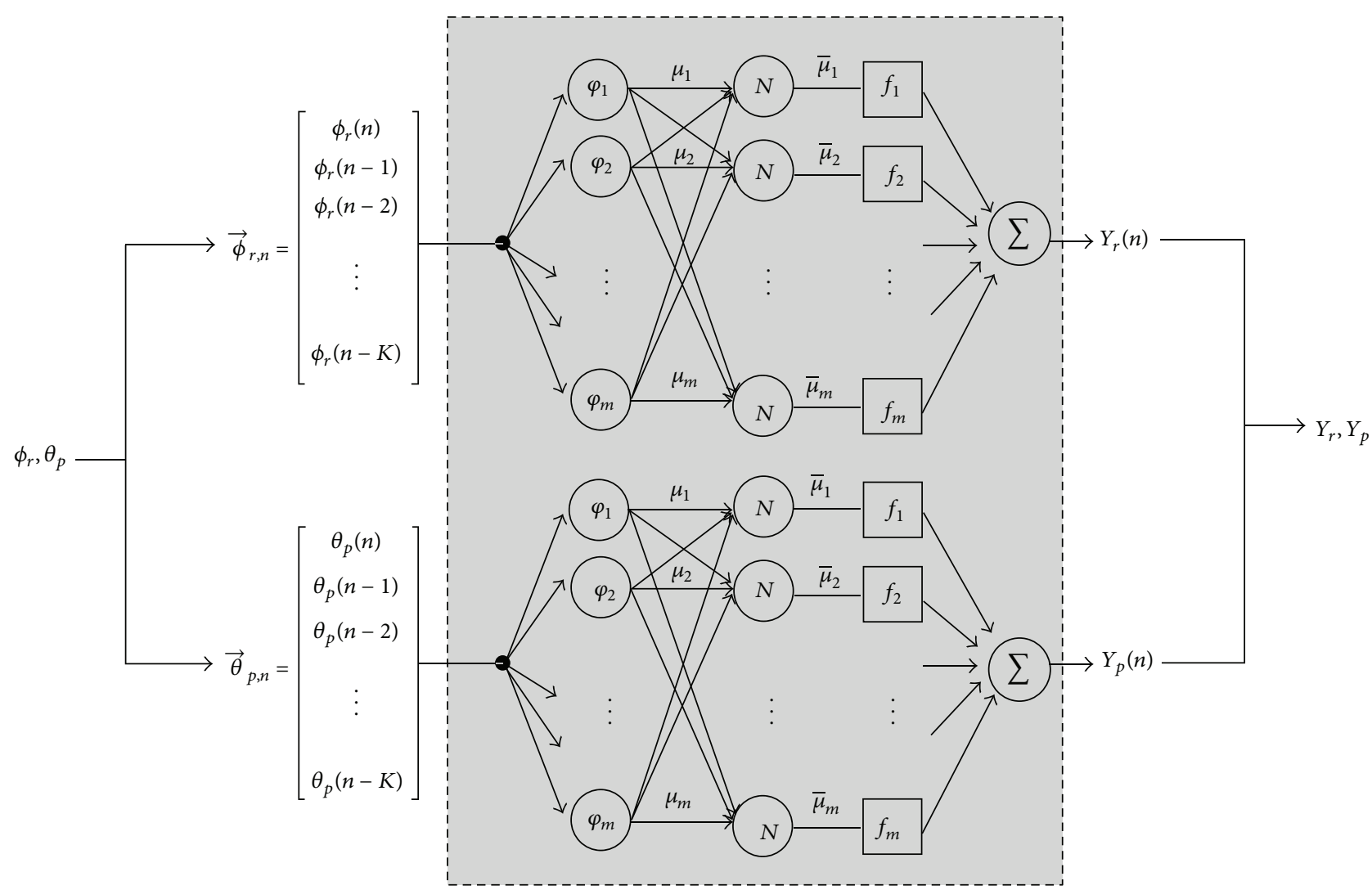

Figure 5: Parallel FBFN beam pointing error estimator.

$$
\mathbf{W}=\left[\begin{array}{cccccccc}
\vec{\mu}_{1, n} A(n) & \vec{\mu}_{1, n} A(n-1) & \cdots & \vec{\mu}_{1, n} A(n-K) & \vec{\mu}_{2, n} & \vec{\mu}_{2, n} A(n) & \cdots & \vec{\mu}_{m, n} \\
\vec{\mu}_{1, n+1} A(n+1) & \vec{\mu}_{1, n+1} A(n) & \cdots & \vec{\mu}_{1, n+1} A(n-K+1) & \vec{\mu}_{2, n+1} & \vec{\mu}_{2, n+1} A(n+1) & \cdots & \vec{\mu}_{m, n+1} \\
\vdots & \vdots & \vdots & \vdots & \vdots & \vdots & \vdots & \vdots \\
\vec{\mu}_{1, n+q-1} A(n+q-1) & \vec{\mu}_{1, n+q-1} A(n+q-2) & \cdots & \vec{\mu}_{1, n+q-1} A(n+q-K-1) & \vec{\mu}_{2, n+q-1} & \vec{\mu}_{2, n+q-1} A(n+q-1) & \cdots & \vec{\mu}_{m, n+q-1}
\end{array}\right]_{q \times(m(K+2))}
$$

Each input training vector correspond to one row in the matrix, which contains $m *(K+2)$ data elements. Therefore, $q$ must be greater than or equal to $m *(K+2)$ in order to solve the consequent parameters. The vector of the desired output in fuzzy inference rules is defined as

$$
\vec{Y}=[y(n), y(n+1), y(n+2), \ldots, y(n+q-1)]^{T} .
$$

A reinforcement learning scheme termed the least-square estimator (LSE) is used to optimally adjust $f_{i}$ in layer three for approximating the desired output of FBFN beam pointing error estimator. The consequent parameter vector is trained as

$$
\widehat{\vec{\alpha}}=\left(\mathbf{W}^{T} \mathbf{W}\right)^{-1} \mathbf{W} \vec{Y} \text {. }
$$

\section{Adaptive Extended Kalman Filter [4]}

The AEKF-based target tracking algorithm $[11,12]$ is used by shipborne phased array radar to recursively update the linear and nonlinear target trajectories and it further improves its tracking accuracy. The distance between the flying target and radar at a time point $t_{j}$ is represented as

$$
r\left(x_{j}, y_{j}, z_{j}\right)=\sqrt{\left(x_{j}-x_{r}\right)^{2}+\left(y_{j}-y_{r}\right)^{2}+\left(z_{j}-z_{r}\right)^{2}},
$$

where $\left(x_{r}, y_{r}, z_{r}\right)$ and $\left(x_{j}, y_{j}, z_{j}\right)$ are the coordinates of radar and air target, respectively. As shown in Figure 3, in polar coordinates, the horizontal angle is expressed as

$$
\phi=\tan ^{-1}\left(\frac{y_{j}-y_{r}}{x_{j}-x_{r}}\right) .
$$




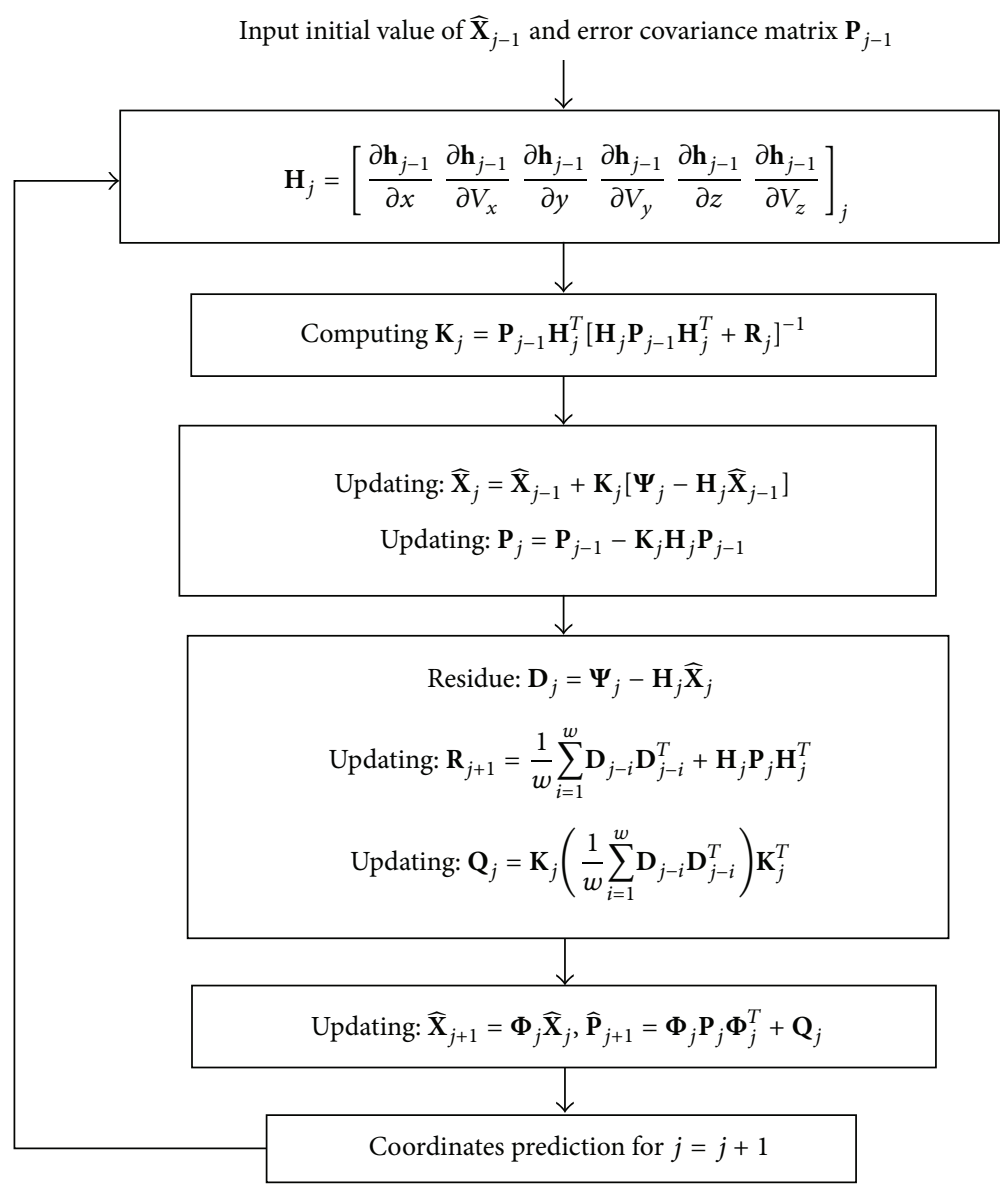

FIGURE 6: The flow chart of AEKF algorithm.

The vertical angle is expressed as

$$
\theta=90^{\circ}-\tan ^{-1}\left(\frac{z_{j}-z_{r}}{\sqrt{\left(x_{j}-x_{r}\right)^{2}+\left(y_{j}-y_{r}\right)^{2}}}\right) .
$$

The system state equation in matrix form is

$$
\mathbf{X}_{j+1}=\Phi_{j} \mathbf{X}_{j}+\mathbf{w}_{j}
$$

where $\mathbf{X}_{j}=\left[\begin{array}{lllllll}x & V_{x} & y & V_{y} & z & V_{z}\end{array}\right]_{j}^{T}$ is the target state vector, where $x_{j}, y_{j}, V_{x, j}, V_{y, j}$ are the relative position of the target in the $x$ - and $y$-axes and relative velocity in the $x$ - and $y$ axes, respectively. The relative acceleration in the $x$ - and $y$ axes is defined as modeling error $\mathbf{w}_{j}$, which is assumed to be random with white Gaussian noise (WGN). $\Delta t=t_{j}-t_{j-1}$ is the detection cycle time. The transition matrix is

$$
\Phi_{j}=\left[\begin{array}{cccccc}
1 & \Delta t & 0 & 0 & 0 & 0 \\
0 & 1 & 0 & 0 & 0 & 0 \\
0 & 0 & 1 & \Delta t & 0 & 0 \\
0 & 0 & 0 & 1 & 0 & 0 \\
0 & 0 & 0 & 0 & 1 & \Delta t \\
0 & 0 & 0 & 0 & 0 & 1
\end{array}\right] .
$$

The measurement equation in matrix form only considers the position measurements in $r, \theta$, and $\phi$, which are not linear equation as

$$
\begin{gathered}
\mathbf{z}_{j}=\mathbf{h}_{j} \mathbf{X}_{j}+\mathbf{v}_{j}, \\
\mathbf{h}_{j} \mathbf{X}_{j}=\left[\begin{array}{lll}
r & \phi & \theta
\end{array}\right]_{j}^{T},
\end{gathered}
$$

where $\mathbf{v}_{j}$ is assumed to be zero mean WGN. The nonlinear equation (32) is linearized by using the first-order Taylor approximation as

$$
\mathbf{z}_{j} \approx \mathbf{z}_{j-1}+\mathbf{H}_{j} \mathbf{X}_{j}-\mathbf{H}_{j} \mathbf{X}_{j-1}+\mathbf{v}_{j},
$$

where the measurement matrix is

$$
\mathbf{H}_{j}=\left[\begin{array}{llllll}
\frac{\partial r}{\partial x} & \frac{\partial r}{\partial V x} & \frac{\partial r}{\partial y} & \frac{\partial r}{\partial V y} & \frac{\partial r}{\partial z} & \frac{\partial r}{\partial V z} \\
\frac{\partial \phi}{\partial x} & \frac{\partial \phi}{\partial V x} & \frac{\partial \phi}{\partial y} & \frac{\partial \phi}{\partial V y} & \frac{\partial \phi}{\partial z} & \frac{\partial \phi}{\partial V z} \\
\frac{\partial \theta}{\partial x} & \frac{\partial \theta}{\partial V x} & \frac{\partial \theta}{\partial y} & \frac{\partial \theta}{\partial V y} & \frac{\partial \theta}{\partial z} & \frac{\partial \theta}{\partial V z}
\end{array}\right]
$$

From (34), the linear measurement equation is redefined as

$$
\Psi_{j} \approx \mathbf{H}_{j} \mathbf{X}_{j}+\mathbf{v}_{j}
$$




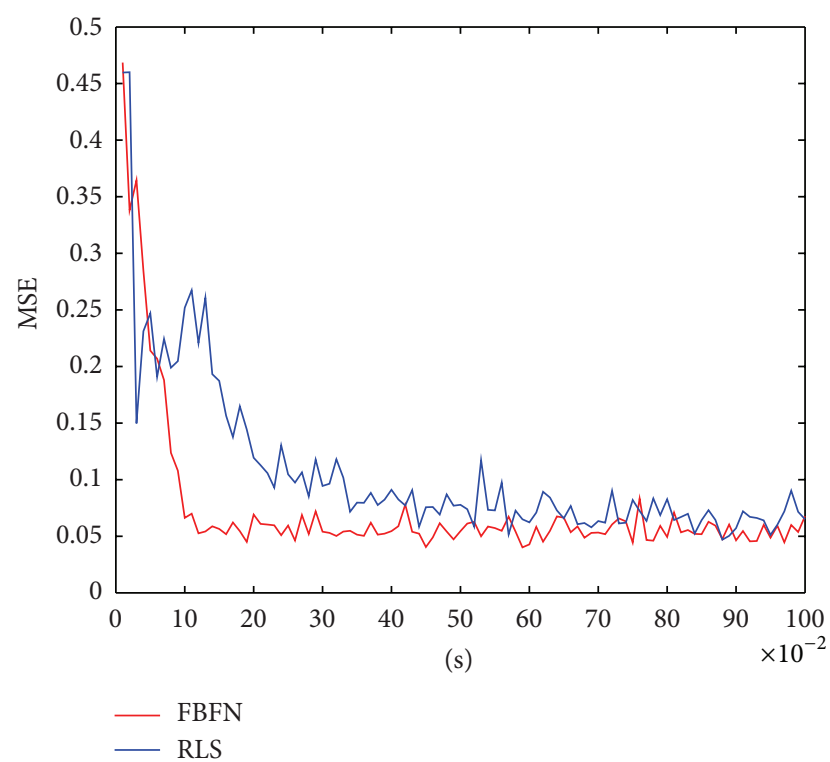

FIGURE 7: Beam pointing error correction for roll angle.

where

$$
\begin{aligned}
& \frac{\partial r}{\partial x}=\left(x_{j-1}-x_{r}\right) \\
& \times\left(\sqrt{\left(x_{j-1}-x_{r}\right)^{2}+\left(y_{j-1}-y_{r}\right)^{2}+\left(z_{j-1}-z_{r}\right)^{2}}\right)^{-1}, \\
& \frac{\partial \phi}{\partial x}=\frac{-\left(y_{j-1}-y_{r}\right)}{\left(x_{j-1}-x_{r}\right)^{2}+\left(y_{j-1}-y_{r}\right)^{2}}, \\
& \frac{\partial \theta}{\partial x}=-\left(x_{j-1}-x_{r}\right)\left(z_{j-1}-z_{r}\right) \\
& \times\left(\left[\left(x_{j-1}-x_{r}\right)^{2}+\left(y_{j-1}-y_{r}\right)^{2}+\left(z_{j-1}-z_{r}\right)^{2}\right]\right. \\
& \left.\times \sqrt{\left(x_{j-1}-x_{r}\right)^{2}+\left(y_{j-1}-y_{r}\right)^{2}}\right)^{-1}, \\
& \frac{\partial r}{\partial y}=\frac{\left(y_{j-1}-y_{r}\right)}{\sqrt{\left(x_{j-1}-x_{r}\right)^{2}+\left(y_{j-1}-y_{r}\right)^{2}+\left(z_{j-1}-z_{r}\right)^{2}}} \\
& \frac{\partial \phi}{\partial y}=\frac{\left(x_{j-1}-x_{r}\right)}{\left(x_{j-1}-x_{r}\right)^{2}+\left(y_{j-1}-y_{r}\right)^{2}} \text {, } \\
& \frac{\partial \theta}{\partial y}=-\left(y_{j-1}-y_{r}\right)\left(z_{j-1}-z_{r}\right) \\
& \times\left(\left[\left(x_{j-1}-x_{r}\right)^{2}+\left(y_{j-1}-y_{r}\right)^{2}+\left(z_{j-1}-z_{r}\right)^{2}\right]\right. \\
& \left.\times \sqrt{\left(x_{j-1}-x_{r}\right)^{2}+\left(y_{j-1}-y_{r}\right)^{2}}\right)^{-1},
\end{aligned}
$$

$$
\begin{aligned}
\frac{\partial r}{\partial z} & =\frac{\left(z_{j-1}-z_{r}\right)}{\sqrt{\left(x_{j-1}-x_{r}\right)^{2}+\left(y_{j-1}-y_{r}\right)^{2}+\left(z_{j-1}-z_{r}\right)^{2}}} \\
\frac{\partial \theta}{\partial z} & =\frac{\sqrt{\left(x_{j-1}-x_{r}\right)^{2}+\left(y_{j-1}-y_{r}\right)^{2}}}{\left[\left(x_{j-1}-x_{r}\right)^{2}+\left(y_{j-1}-y_{r}\right)^{2}+\left(z_{j-1}-z_{r}\right)^{2}\right]} \\
\frac{\partial r}{\partial V x} & =\frac{\partial r}{\partial V y}=\frac{\partial r}{\partial V z}=\frac{\partial \theta}{\partial V x}=\frac{\partial \theta}{\partial V y}=\frac{\partial \theta}{\partial V z} \\
& =\frac{\partial \phi}{\partial V x}=\frac{\partial \phi}{\partial V y}=\frac{\partial \phi}{\partial V z}=\frac{\partial \phi}{\partial z}=0 .
\end{aligned}
$$

The covariance matrix of process noise is defined as

$$
\mathbf{Q}_{j}=E\left\{\mathbf{w}_{j} \mathbf{w}_{j}^{T}\right\} .
$$

The covariance matrix of measurement noise is defined as

$$
\mathbf{R}_{j}=E\left\{\mathbf{v}_{j} \mathbf{v}_{j}^{T}\right\} .
$$

Due to the impact of ship movement and environmental noise, process noise and measurement noise will dynamically change, so that the covariance matrix values of process noise and measurement noise are adaptively estimated to ensure the extended Kalman filter convergence. The covariance matrix of measurement noise is obtained with

$$
\mathbf{R}_{j+1}=\widehat{\mathbf{C}}_{v}+\mathbf{H}_{j} \mathbf{P}_{j} \mathbf{H}_{j}^{T}
$$

where

$$
\begin{aligned}
\widehat{\mathbf{C}}_{v} & =\frac{1}{w} \sum_{i=1}^{w}\left(\boldsymbol{\Psi}_{j-i}-\mathbf{H}_{j-i} \widehat{\mathbf{X}}_{j-i}\right)\left(\boldsymbol{\Psi}_{j-i}-\mathbf{H}_{j-i} \widehat{\mathbf{X}}_{j-i}\right)^{T} \\
& =\frac{1}{w} \sum_{i=1}^{w} \mathbf{D}_{j-i} \mathbf{D}_{j-i}{ }^{T}
\end{aligned}
$$

where the residual value $\mathbf{D}_{j}$ is the difference between measurement and estimation, and $w$ is the moving window size. The covariance matrix of process noise is obtained with

$$
\mathbf{Q}_{j}=\mathbf{K}_{j} \widehat{\mathbf{C}}_{v} \mathbf{K}_{j}^{T}
$$

The flow chart of AEKF algorithm is shown in Figure 6.

\section{Computer Simulations}

Six scenarios are used to simulate the tracking performance of shipborne phased array radar using the proposed beam pointing error compensation mechanism.

Case 1 (beam pointing error compensation for stationary ship and target). The ship's roll and pitch signals are simulated according to (3) and (4) with roll and pitch angle parameters of sea state 5 listed in Table 1 and standard deviation of 0.05 . 
The beam of $6 \times 6$ planar array antenna is steered to $(\theta, \phi)=\left(45^{\circ}, 60^{\circ}\right)$. The sampling frequency is set as $100 \mathrm{~Hz}$. Therefore, the ship's roll and pitch signals are sampled 1000 points within 10 seconds, which is used as the center value of 1000 membership functions for parallel beam pointing error estimator. The simulated ship's roll and pitch signals are applied for the FBFN architecture and the RLS adaptive prediction filter to simulate the performance of pointing error estimation. Each of the ship's roll and pitch signals is sampled 13 points for each of simulations. The simulation replicates 1000 times. The input samples are updated by one new sample for the next iteration. Figures 7 and 8 compare the ensemble-averaged square error performance of the FBFN architecture and RLS adaptive prediction filter for roll and pitch angles, respectively, per $0.01 \mathrm{sec}$ iteration time. Table 2 compares the convergent values of ensemble-averaged square beam pointing error for FBFN architecture and RLS adaptive prediction filter under different sea states and standard deviation of 0.05 . The ensemble-averaged square error is defined as

$$
\operatorname{MSE}(n)=\frac{1}{1000} \sum_{m=1}^{1000}\left[\theta_{m}(n+1)-Y_{m}(n)\right]^{2},
$$

where $m$ represents the $m$ th Monte-Carlo simulation; $n=$ $1, \ldots, 100$ represents the number of the iterations.

Based on the simulation results, we may make the following observations. The FBFN architecture converges much faster than the RLS adaptive prediction filter and it has lower ensemble-averaged square error than RLS adaptive prediction filter. The steady state values of the ensemble-averaged square error for both the FBFN architecture and RLS adaptive prediction filter approximate to the standard deviation of the Gaussian noise. In summary, the convergent time and accuracy of the automatic beam error correction system using the FBFN architecture is superior to the RLS adaptive prediction filter. The shortest convergent time is $0.1 \mathrm{sec}$ when FBFN beam pointing error estimator is used (see Table 3).

Case 2 (beam pointing error estimation and compensation for linear moving ship and target). Figures 9(a) and 9(b) shows the simulation scenario of beam pointing error compensation for linear moving air target and ship with linear motion. Assuming the ship is along the $Y$-axis of $X Y$ plane in the earth coordinates, the ship speed is $10 \mathrm{~m} / \mathrm{sec}$ and the flying target speed is $300 \mathrm{~m} / \mathrm{sec}$. The flying target is parallel to the $X Y$ plane, $9.84 \mathrm{~km}$ above the ground, and the angle between the flight direction and the $Y$-axis is $30^{\circ}$. The largest reconnaissance distance of shipborne radar is $150 \mathrm{~km}$. while the air target is flying within the radar detection range, the beam pointing error estimation and compensation of shipborne phased array radar are simulated.

The linear ship path equation is

$$
w s_{t}\left(x_{t}, y_{t}, z_{t}\right)=w s_{0}+v_{w s} t
$$

where $t=1,2,3, \ldots, 1010,1011 \mathrm{sec}, v_{w s}$ is ship velocity, and $w s_{0}$ is initial ship position. The linear target trajectory equation is

$$
m s_{t}\left(x_{t}, y_{t}, z_{t}\right)=m s_{0}+v_{m s} t
$$

TABLE 2: Comparison of the convergent values for different sea states.

\begin{tabular}{lcccccc}
\hline & \multicolumn{9}{c}{ Sea state } \\
Algorithm & \multicolumn{2}{c}{ Sea 4} & \multicolumn{2}{c}{ Sea 5 } & \multicolumn{2}{c}{ Sea 6} \\
& & \multicolumn{2}{c}{ Angle } \\
& Roll & Pitch & Roll & Pitch & Roll & Pitch \\
\hline No correction & 2.53 & 0.21 & 43.90 & 3.01 & 90.49 & 9.96 \\
RLS & 0.062 & 0.059 & 0.067 & 0.061 & 0.071 & 0.064 \\
FBFN & 0.055 & 0.081 & 0.056 & 0.056 & 0.057 & 0.059 \\
\hline
\end{tabular}

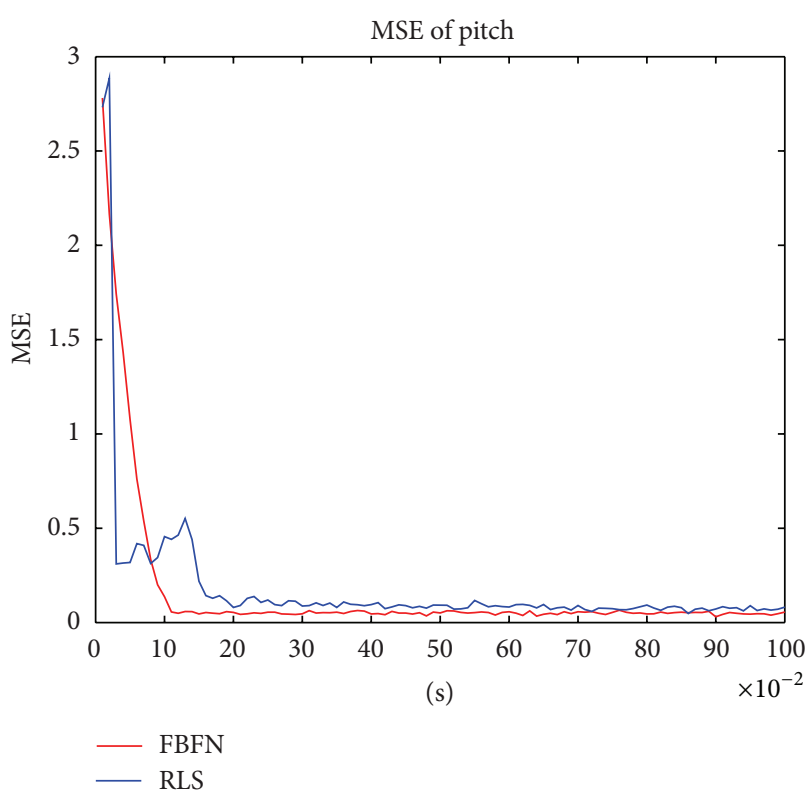

FIGURE 8: Beam pointing error correction for pitch angle.

where $v_{m s}$ is the target velocity, and $m s_{0}$ is the initial target position.

Figures 10(a) and 10(b) compare the beam pointing error compensation performance of the FBFN architecture, RLS adaptive prediction filter for roll and pitch angles, respectively, under the sea state 5 condition. The green curve represents the real beam steering angle of the shipborne phased array radar. The initial beam angle is steered to $(\theta, \phi)=$ $\left(70^{\circ}, 150^{\circ}\right)$, and the following beam angles are calculated by (20) and (21). The FBFN architecture converges faster than the RLS adaptive prediction filter. The peak response of the RLS adaptive prediction filter will result in the performance degradation of the shipborne phased array radar.

Case 3 (beam pointing error estimation and compensation for linear moving ship and circular trajectory target). Figures 11(a) and 11(b) show the simulation scenario of beam pointing error compensation for circular maneuvering air target and linear moving ship. The ship has the same linear moving speed and path equation. The flying target is parallel to the $X Y$ plane, $9.84 \mathrm{~km}$ above the ground, and the angle between the initial position and the $Y$-axis is $30^{\circ}$. The trajectory of the equal speed flight vehicle is a circle with a radius of $10 \mathrm{~km}$, and 


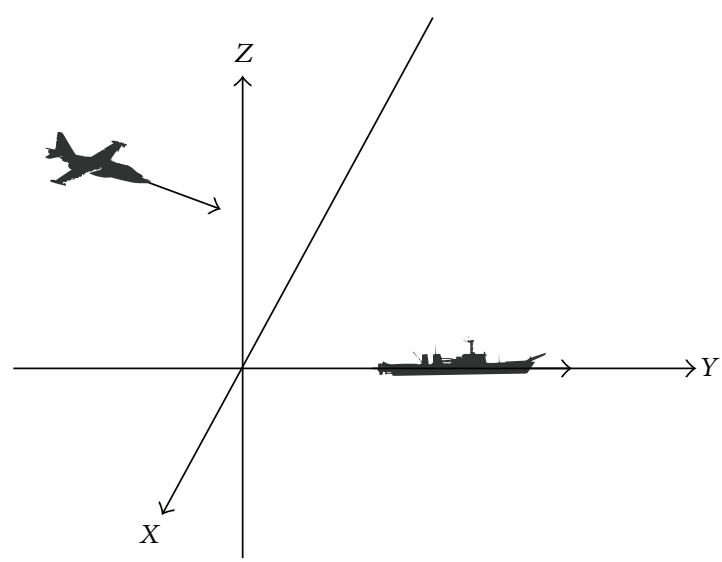

(a)

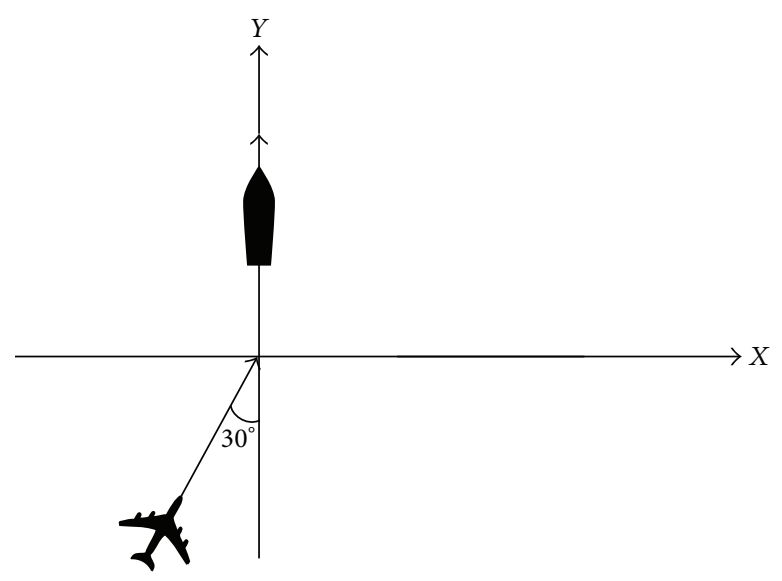

(b)

Figure 9: Simulation scenarios of Case 2 for (a) 3D and (b) top view diagrams.

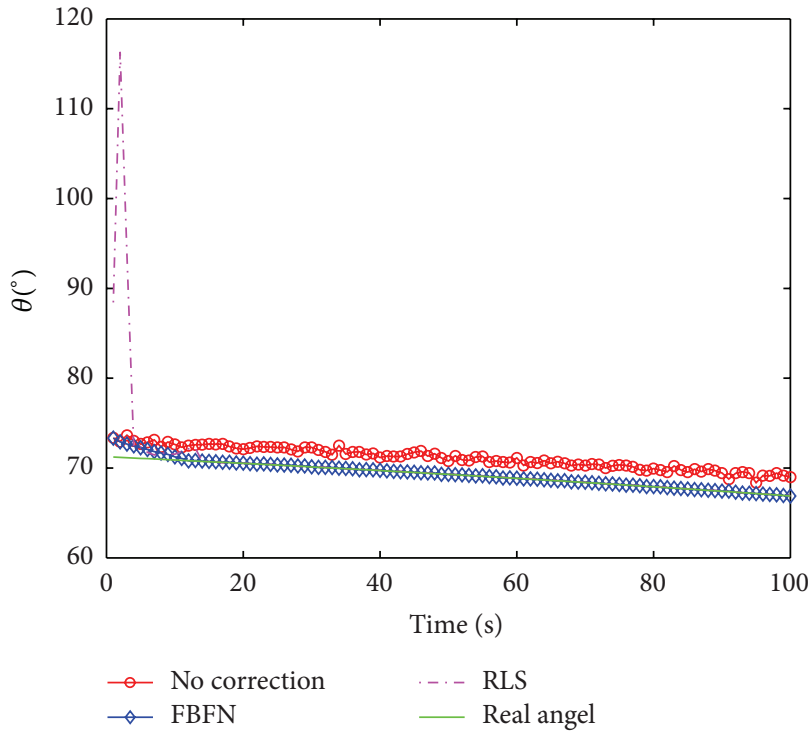

(a)

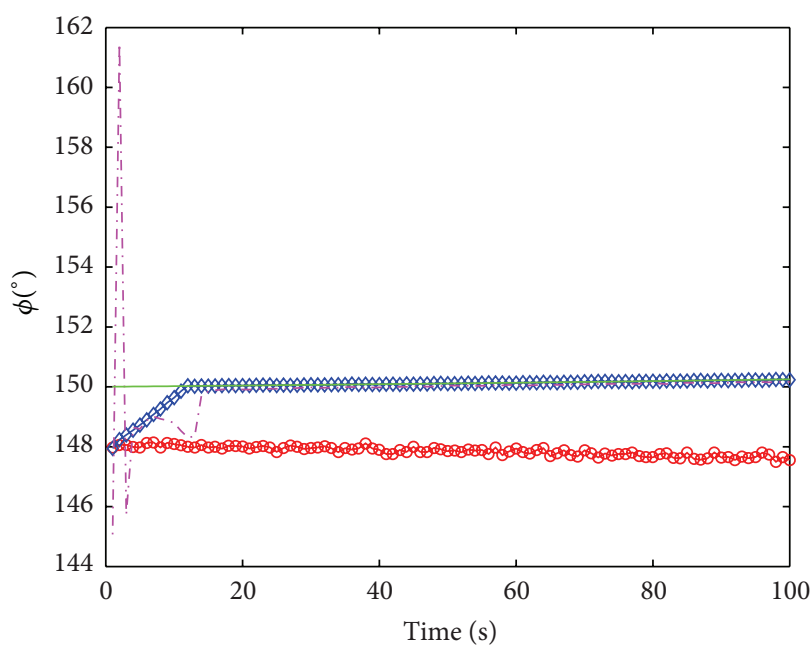

$\begin{array}{ll}\rightarrow \text { No correction } & \cdots \\ \rightarrow \text { FBFN } & \text { RLS } \\ & \quad \text { Real angel }\end{array}$

(b)

FIGURE 10: (a) Elevation and (b) horizontal beam pointing error compensation for linear moving target.

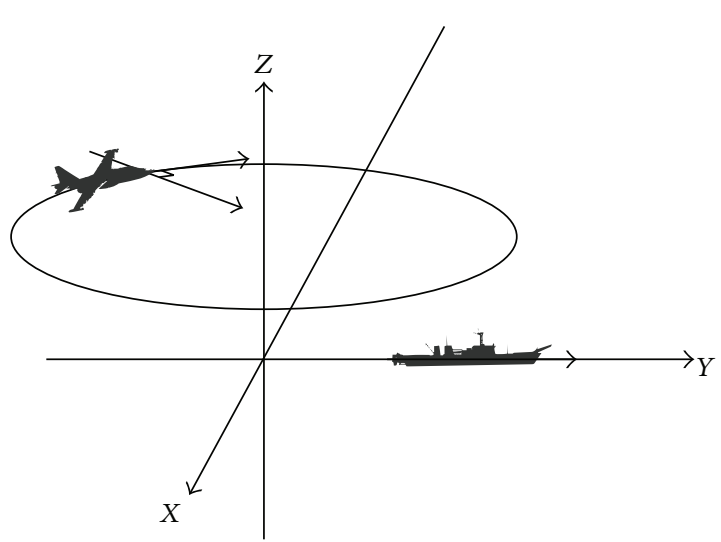

(a)

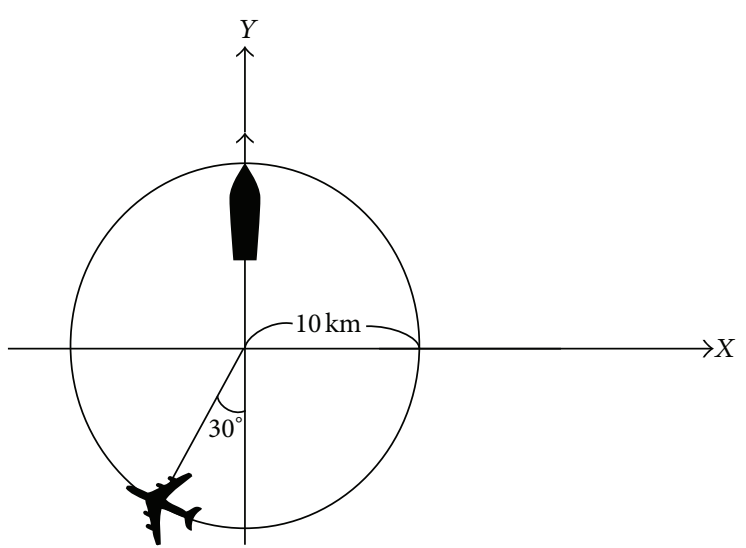

(b)

Figure 11: Simulation scenarios of Case 3 for (a) 3D and (b) top view diagrams. 
TABLE 3: Comparison of the convergent values for different standard deviations.

\begin{tabular}{|c|c|c|c|c|c|c|c|c|c|c|}
\hline \multirow{3}{*}{ Algorithm } & \multicolumn{10}{|c|}{ Standard deviation } \\
\hline & \multicolumn{2}{|c|}{0.01} & \multicolumn{2}{|c|}{0.05} & \multicolumn{2}{|c|}{$\begin{array}{c}0.1 \\
\text { Angle }\end{array}$} & \multicolumn{2}{|c|}{0.5} & \multicolumn{2}{|c|}{1} \\
\hline & Roll & Pitch & Roll & Pitch & Roll & Pitch & Roll & Pitch & Roll & Pitch \\
\hline No correction & 43.831 & 2.9585 & 43.903 & 3.0071 & 43.956 & 3.0428 & 44.404 & 3.4406 & 44.971 & 3.9836 \\
\hline RLS & 0.0145 & 0.0127 & 0.0674 & 0.0608 & 0.1292 & 0.1291 & 0.6479 & 0.6357 & 1.3071 & 1.2154 \\
\hline FBFN & 0.0113 & 0.0105 & 0.0556 & 0.0555 & 0.1122 & 0.1072 & 0.5607 & 0.5239 & 1.1057 & 1.4081 \\
\hline
\end{tabular}

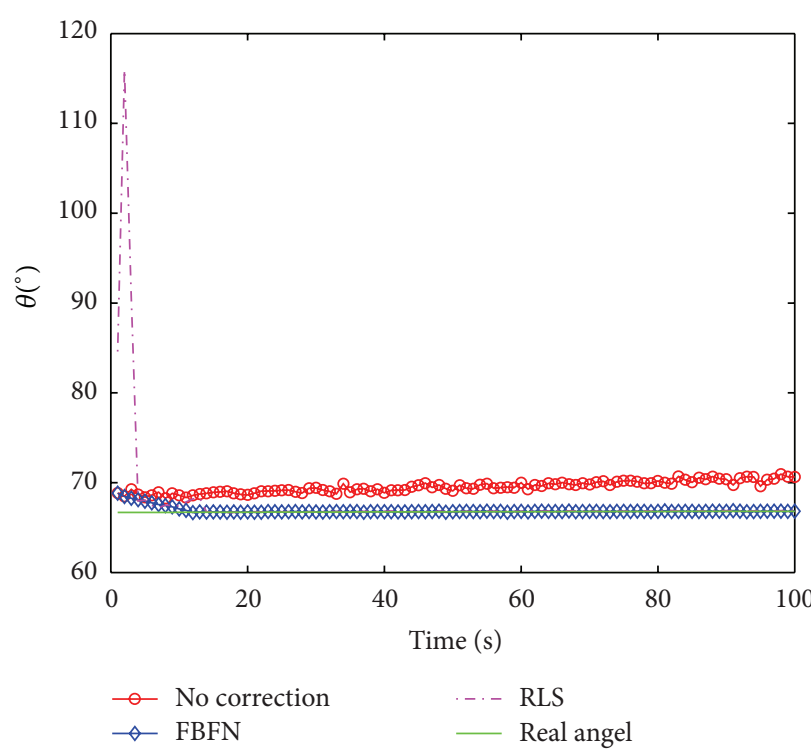

(a)

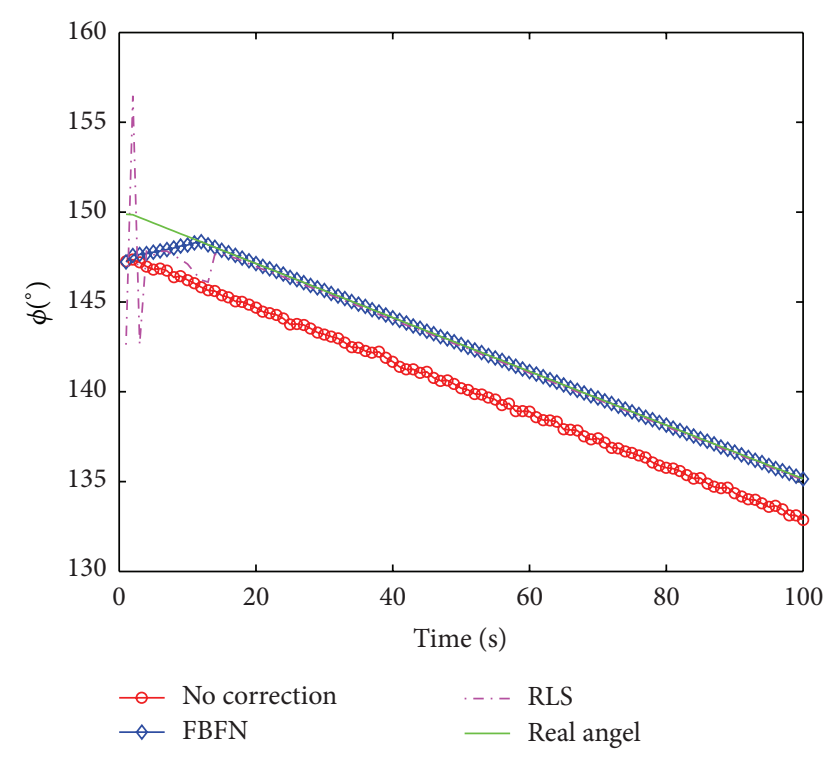

(b)

FIGURE 12: (a) Elevation and (b) horizontal beam pointing error compensation for circular maneuvering target.

the $Y$-axis is the center. The rotational cycle time of the flight target is 209 seconds.

The circular trajectory equation is

$$
m s_{t}\left(x_{t}, y_{t}, z_{t}\right)=\left(r \cos \left(\theta_{0}-\omega t\right), r \sin \left(\theta_{0}-\omega t\right), 9.84\right) \text {, }
$$

where $t=1,2,3, \ldots, 418,419 \mathrm{sec}, r$ is the circular flight radius, $\theta_{0}$ is the initial angle between the flight vehicle and the $X$-axis, and $\omega$ is the angular speed of flight vehicle.

When the vehicle flies in circles with constant speed, the simulation results for roll and pitch angles are shown in Figures 12(a) and 12(b), respectively. The FBFN architecture converges faster than the RLS adaptive prediction filter and compensates the beam pointing error effectively.

Case 4 (AEKF with no beam pointing error for linear trajectory target). Assuming the ship is not affected by the sea waves (antenna beam pointing error is zero), the tracking performance of AEKF for a straight flight target trajectory is simulated. As shown in Figure 13, the initial position of radar is $(0,0,0)$. The ship moves with a speed of $10 \mathrm{~m} / \mathrm{sec}$ in the $Y$ axial direction. The radar position is updated every second. The initial position of flying targets is $-74840,-129620$, and 9100 meters, about $150 \mathrm{~km}$ from the radar. The flight speed of target is $300 \mathrm{~m} / \mathrm{sec}$ ( $X$ axial velocity of $150 \mathrm{~m} / \mathrm{sec}$ and $Y$ axial velocity of $260 \mathrm{~m} / \mathrm{sec}$ ). The target location is updated every second. The tracking accuracy of AEKF for a straight flight target is shown in Figure 14, where the trajectory estimation error is calculated by

$$
E_{j}=\sqrt{\left(x_{j}-\widehat{x}_{j}\right)^{2}+\left(y_{j}-\widehat{y}_{j}\right)^{2}+\left(z_{j}-\widehat{z}_{j}\right)^{2}} .
$$

It shows that the AEKF converges to less than about $20 \mathrm{~m}$ after 550 iterations.

Case 5 (AEKF with no beam pointing error for circular trajectory target). Assuming the ship is not affected by the sea waves (antenna beam pointing error is zero), the tracking performance of AEKF for a circular trajectory target is simulated. As shown in Figure 15, the initial position of radar is $(0,0,0)$. The ship moves with a speed of $10 \mathrm{~m} / \mathrm{sec}$ in the $Y$ axial direction. The radar position is updated every second. The initial position of flying targets is $-5000,-8660$, and 9100 meters about $13.5 \mathrm{~km}$ from the radar. The flying target is parallel to the $X Y$ plane, $9.84 \mathrm{~km}$ above the ground, and the angle between the initial position and the $Y$-axis is $30^{\circ}$. The flying object has uniform velocity and circular trajectory of radius of $10 \mathrm{~km}$, and the $Y$-axis is the center. The rotational 


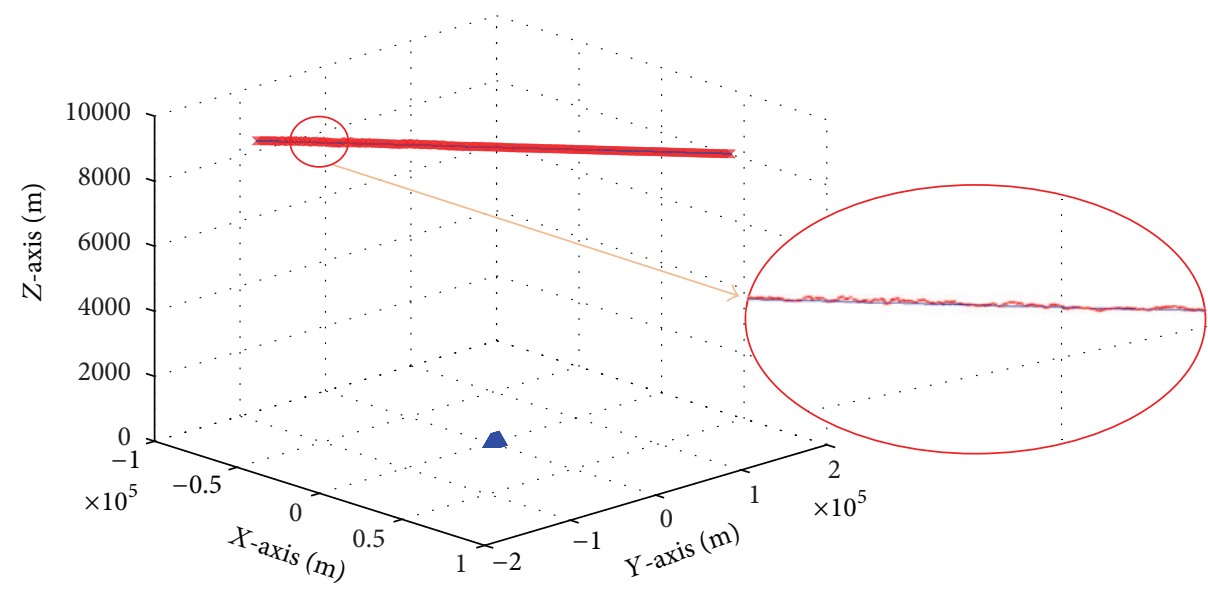

$\Delta \quad$ Radar path

- Real path

$\rightarrow$ Estimation path

FIGURE 13: 3D scenario diagram of Case 4.

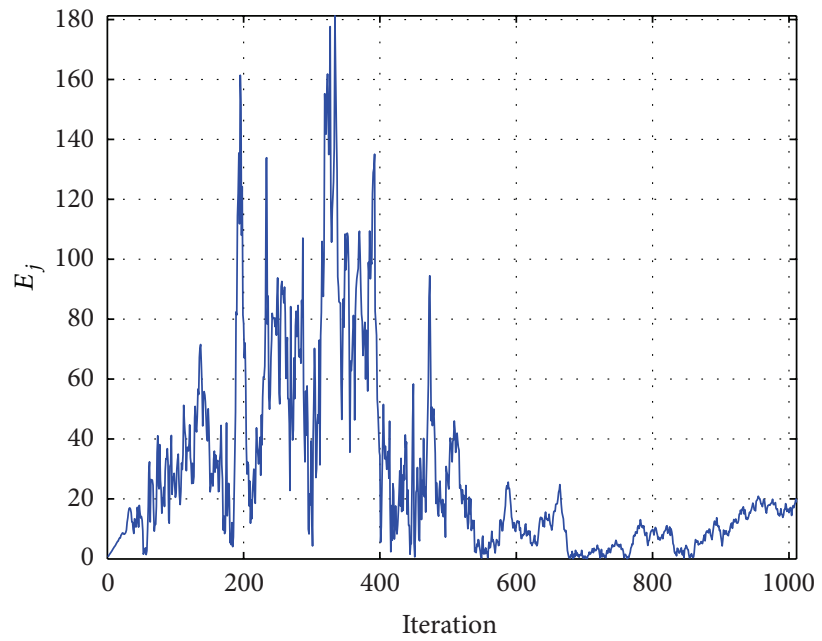

FIGURE 14: Tracking accuracy of AEKF for straight flight target.

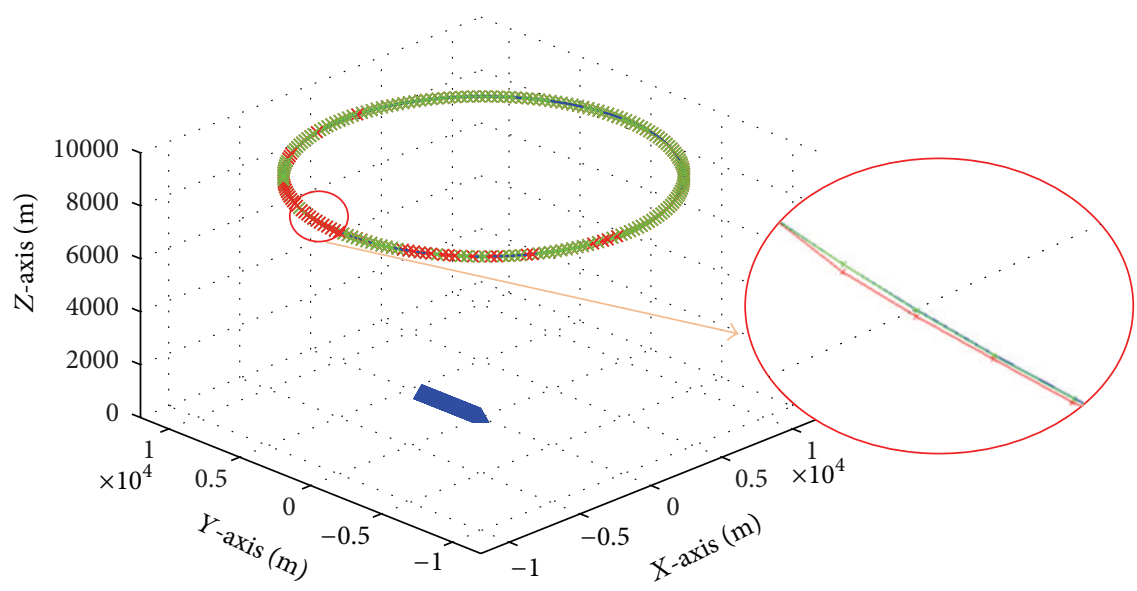

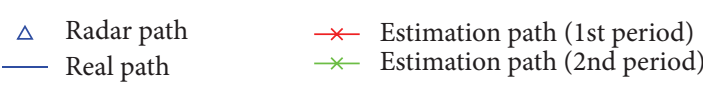

FIGURE 15: 3D scenario diagram of Case 5. 


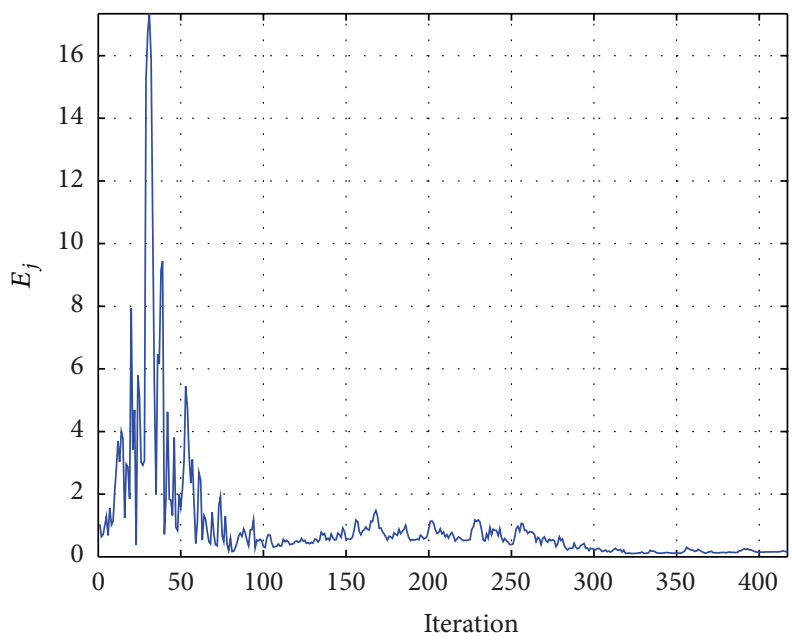

FIGURE 16: Tracking accuracy of AEKF for straight flight target.

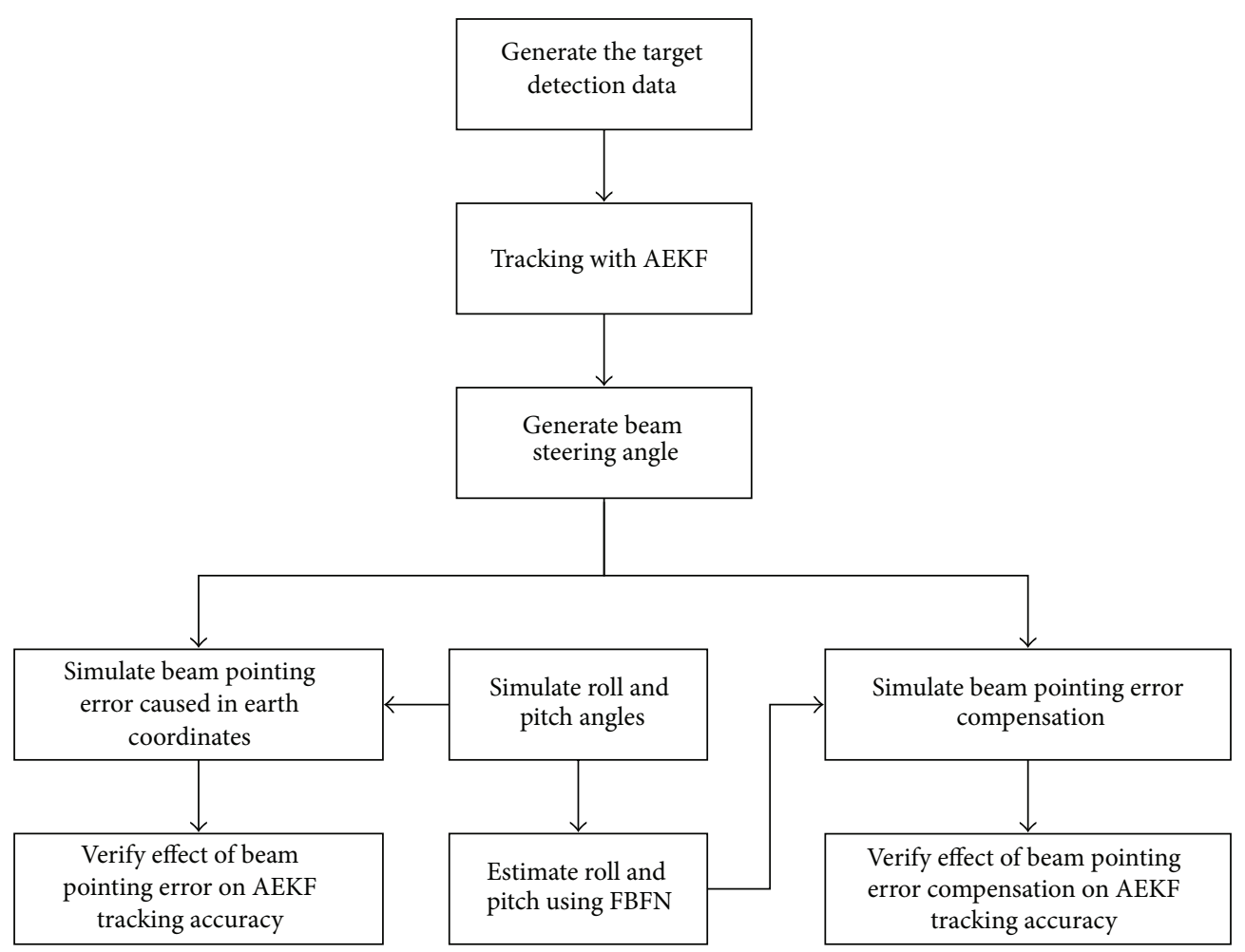

FIGURE 17: Flow chart of simulation used to verify the effect of beam pointing error compensation on AEKF tracking accuracy.

cycle time of the flying target is 209 seconds. The target flies two cycles in the simulation. The tracking accuracy of AEKF for a circular trajectory flight target is shown in Figure 16. The AEKF converges to less than $1 \mathrm{~m}$ after 100 iterations, because the distance between radar and flight target in Case 3 is less than Case 5.

Case 6 (combining AEKF with beam pointing error compensation for linear trajectory target). Figure 17 is a simulation flow chart used to verify the effect of beam pointing error compensation on AEKF tracking accuracy. The shipbone radar and flight target have the same experimental scenario as Case 4, but beam pointing error generated by roll and pitch angle parameters of sea state 5 is considered. The linear trajectory target is shown in Figure 13.

When the beam pointing of shipborne phased array antenna is affected by the roll and pitch angles, the target tracking error of AEKF is shown in Figure 18. It shows that the AEKF converges to less than about $20 \mathrm{~m}$ at 550 iterations but the estimation error increases to $100 \mathrm{~m}$ at 1000 iterations. We conclude that the tracking accuracy is affected by the beam pointing error and the increasing distance between 


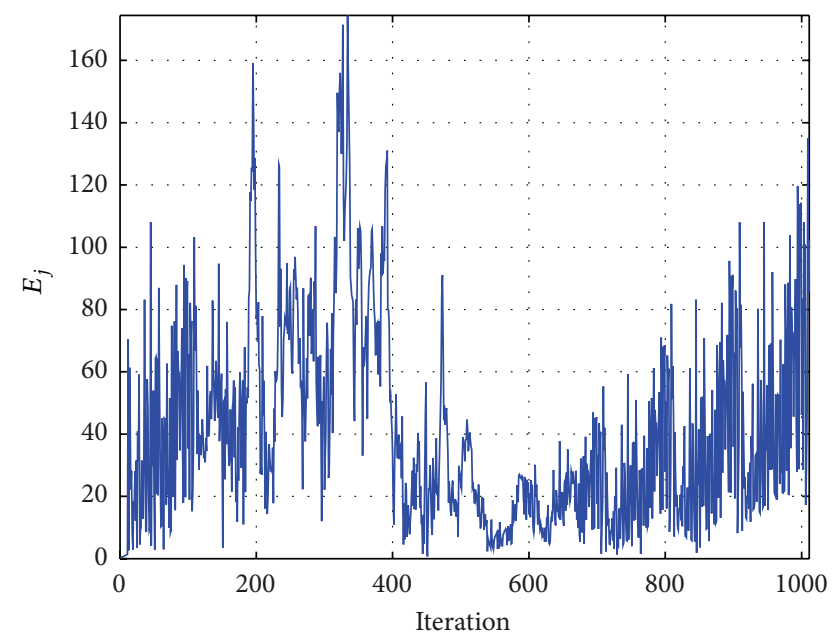

FIGURE 18: Estimation error of AEKF without beam pointing error compensation.

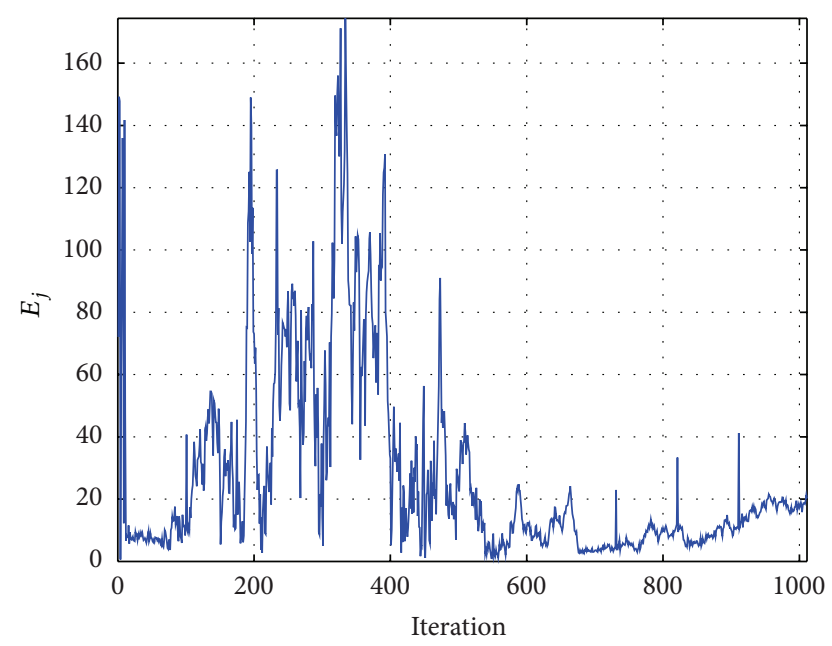

FIGURE 19: Estimation error of AEKF with FBFN based beam pointing error compensation.

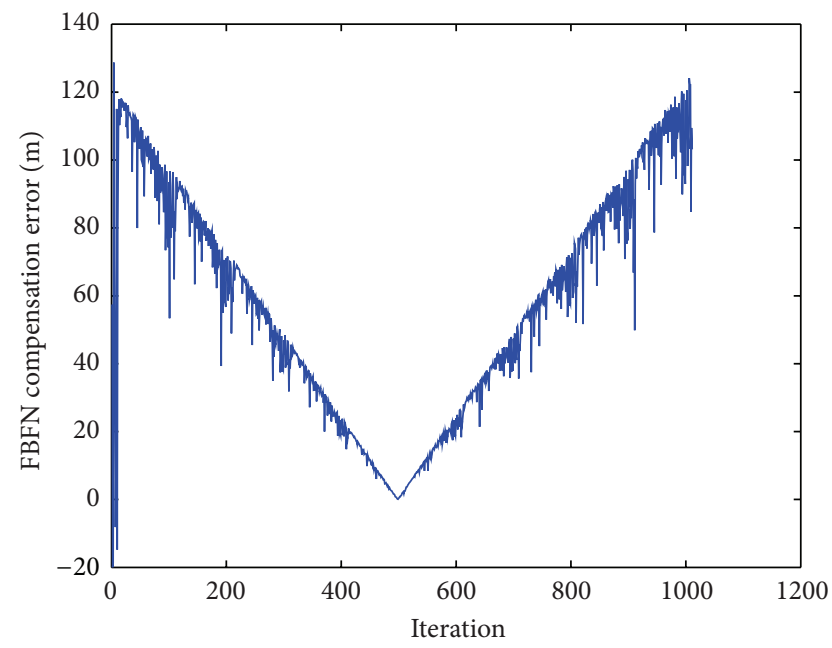

FIGURE 20: Error compensation value of FBFN controller. the radar and flight target obviously when the simulation result of Figure 18 is compared with Figure 14. When the beam pointing error of shipborne phased array antenna is compensated with FBFN controller, the target tracking error of AEKF is shown in Figure 19. It shows that the tracking error of AEKF converges to less than about $20 \mathrm{~m}$ at 550 iterations and the estimation error will remain within the range of about $20 \mathrm{~m}$ when beam pointing error is compensated by FBFN controller. The error compensation values of FBFN controller are shown in Figure 20, which is calculated by subtracting the trajectory estimation error of Figure 18 from Figure 19. The error compensation values of FBFN controller present a V-shaped curve. The tracking performance of AEKF is proportional to the distance between moving target and the ship. In the simulation of the starting point and end point, moving targets far from the shipborne radar, the FBFN controller generates the largest estimation error. When the moving target closes the radar just above the ship, the FBFN controller generates the smallest estimation error.

\section{Conclusions}

This paper proposed an intelligent beam pointing error compensation mechanism for shipborne two-dimensional phase array antenna radar. The FBFN controller estimates the roll and pitch angle of the ship driving in the sea and thus compensates for the antenna beam pointing error in order to enhance the accuracy of phased array radar system tracking a moving target. Six cases of simulations are used to verify the performance of shipborne phased array radar using the proposed beam pointing error compensation mechanism for six different scenarios. Cases 1, 2, and 3 simulate the accuracy of beam pointing error compensation mechanism and Cases 4 , 5 , and 6 simulate the effect of beam pointing error compensation mechanism on the tracking accuracy of AEKF.

The simulation results show that the convergent time and accuracy of the automatic beam pointing error compensation mechanism using the FBFN architecture are superior to the RLS adaptive prediction filter. The tracking accuracy is affected by the beam pointing error and the distance between the radar and moving target. In summary, the use of the proposed FBFN beam pointing error compensation mechanism with the AEKF can reduce the amount of estimation error significantly.

\section{Acknowledgment}

This work is supported in part by research grants from the National Science Council, Taiwan (NSC 102-2218-E-155-001).

\section{References}

[1] Z. Fang and Q. Rundong, "Ship-borne phased array radar motion compensation," in System Engineering and Electronic Technologies, pp. 4-8, 1998.

[2] S. L. Chen, Modeling, dynamics and control of large amplitude motion of vessel in beam seas [Ph.D. thesis], Department of Mechanical Engineering, Michigan State University, 1996. 
[3] L.-X. Wang, "Stable adaptive fuzzy control of nonlinear systems," IEEE Transactions on Fuzzy Systems, vol. 1, no. 2, pp. 146155, 1993.

[4] V. Fathabadi, M. Shahbazian, K. Salahshour, and L. Jargani, "Comparison of adaptive Kalman filter methods in state estimation of a nonlinear system using asynchronous measurements," in Proceedings of the World Congress on Engineering and Computer Science (WCECS '09), vol. 2, pp. 20-22, San Francisco, Calif, USA, October 2009.

[5] D. C. Law, S. A. McLaughlin, M. J. Post et al., "An electronically stabilized phased array system for shipborne atmospheric wind profiling," Journal of Atmospheric and Oceanic Technology, vol. 19, no. 6, pp. 924-933, 2002.

[6] C. A. Balanis, Antenna Theory: Analysis and Design, John Wiley \& Sons, 3rd edition, 2005.

[7] J. Mar and Y.-R. Lin, "Implementation of SDR digital beamformer for microsatellite SAR," IEEE Geoscience and Remote Sensing Letters, vol. 6, no. 1, pp. 92-96, 2009.

[8] S. Haykin, Adaptive Filter Theory, Prentice Hall, 3rd edition, 1996.

[9] E. Mosca, Optimal, Predictive, and Adaptive Control, Prentice Hall, 1995.

[10] Y. Li and Y.-T. Jang, "Complex adaptive fuzzy inference systems," in Proceedings of the Asian Fuzzy Systems Symposium, pp. 551556, December 1996.

[11] A. Almagbile, J. L. Wang, and W. Ding, "Evaluating the performance of adaptive Kalman filter methods in GPS/INS integration," Journal of Global Positioning System, vol. 9, no. 1, pp. 33-40, 2010.

[12] K. H. Kim, J. G. Lee, and C. G. Park, "Adaptive two-stage extended kalman filter for a fault-tolerant INS-GPS loosely coupled system," IEEE Transactions on Aerospace and Electronic Systems, vol. 45, no. 1, pp. 125-137, 2009. 

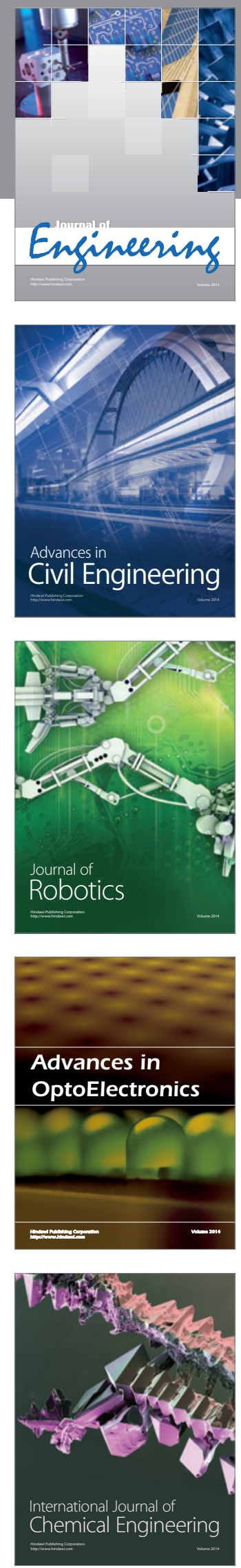

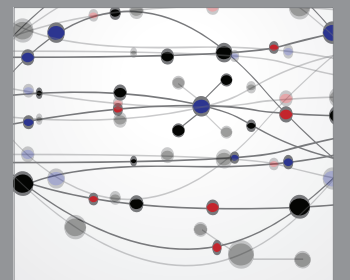

The Scientific World Journal
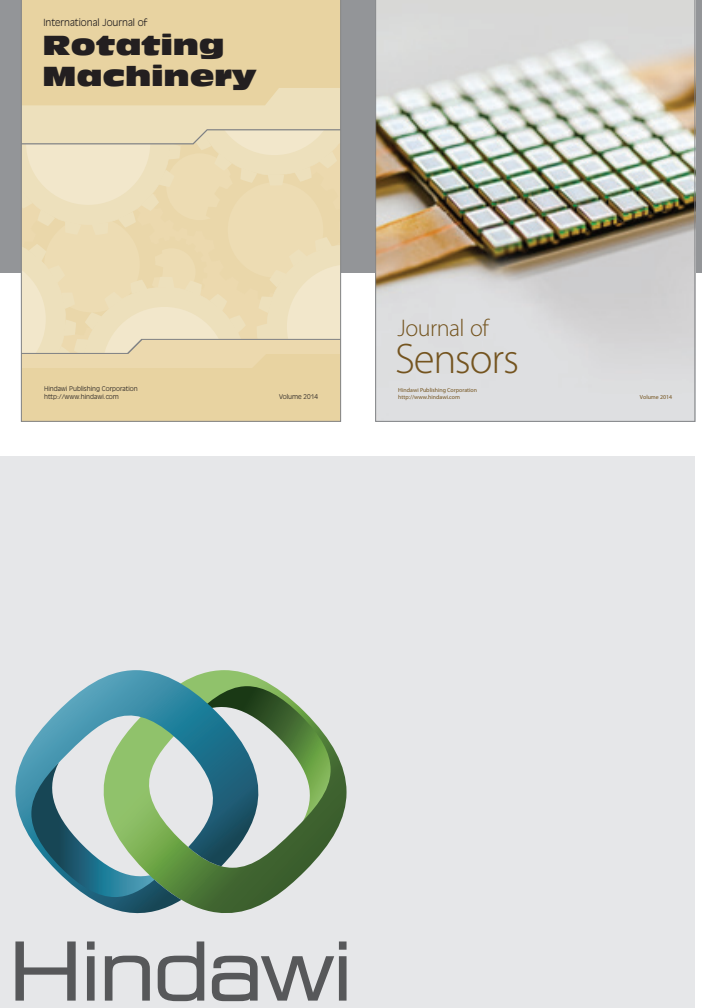

Submit your manuscripts at http://www.hindawi.com
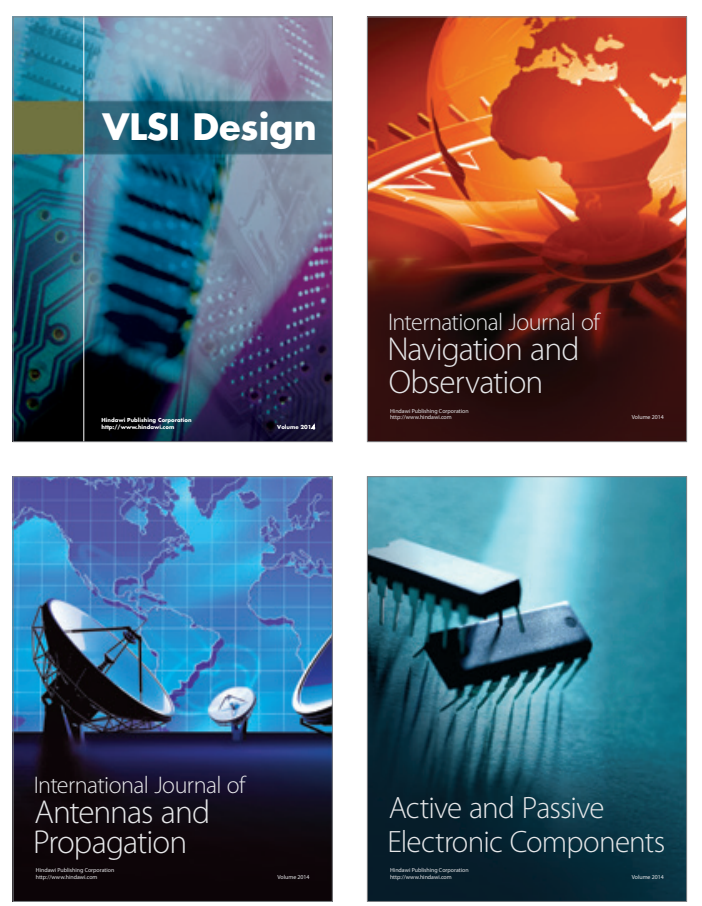
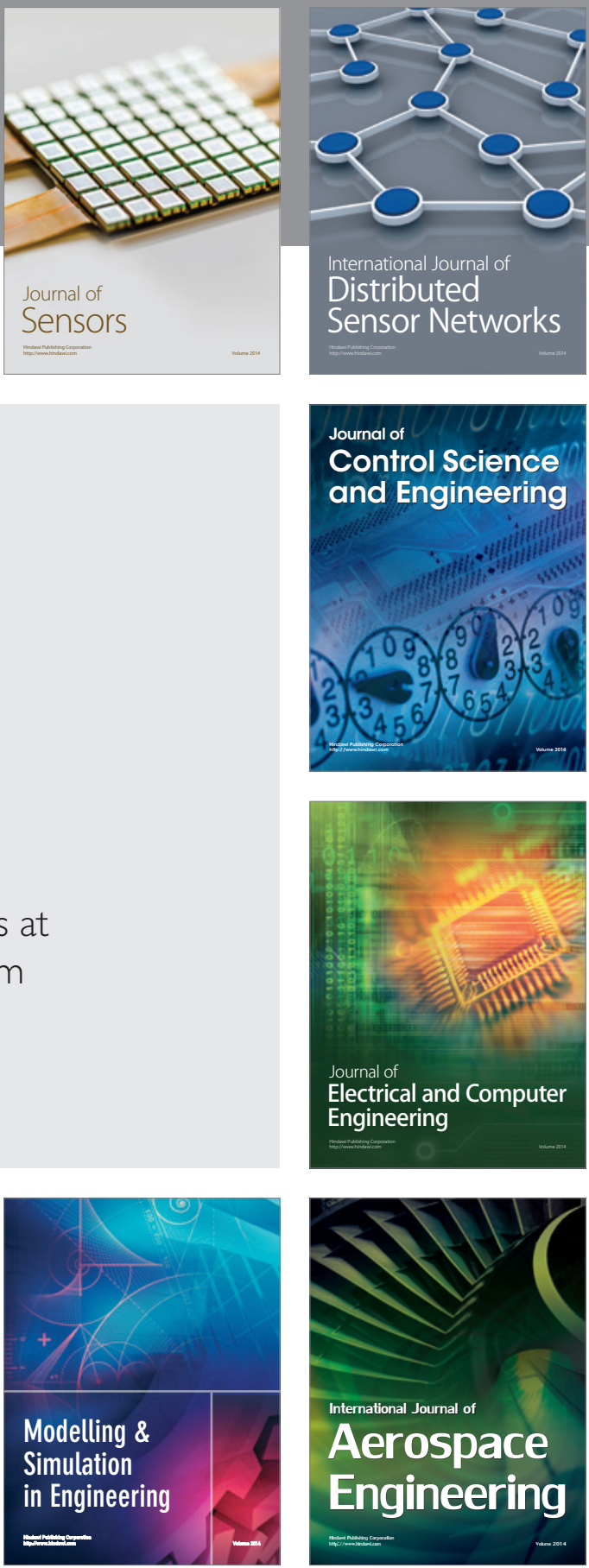

Journal of

Control Science

and Engineering
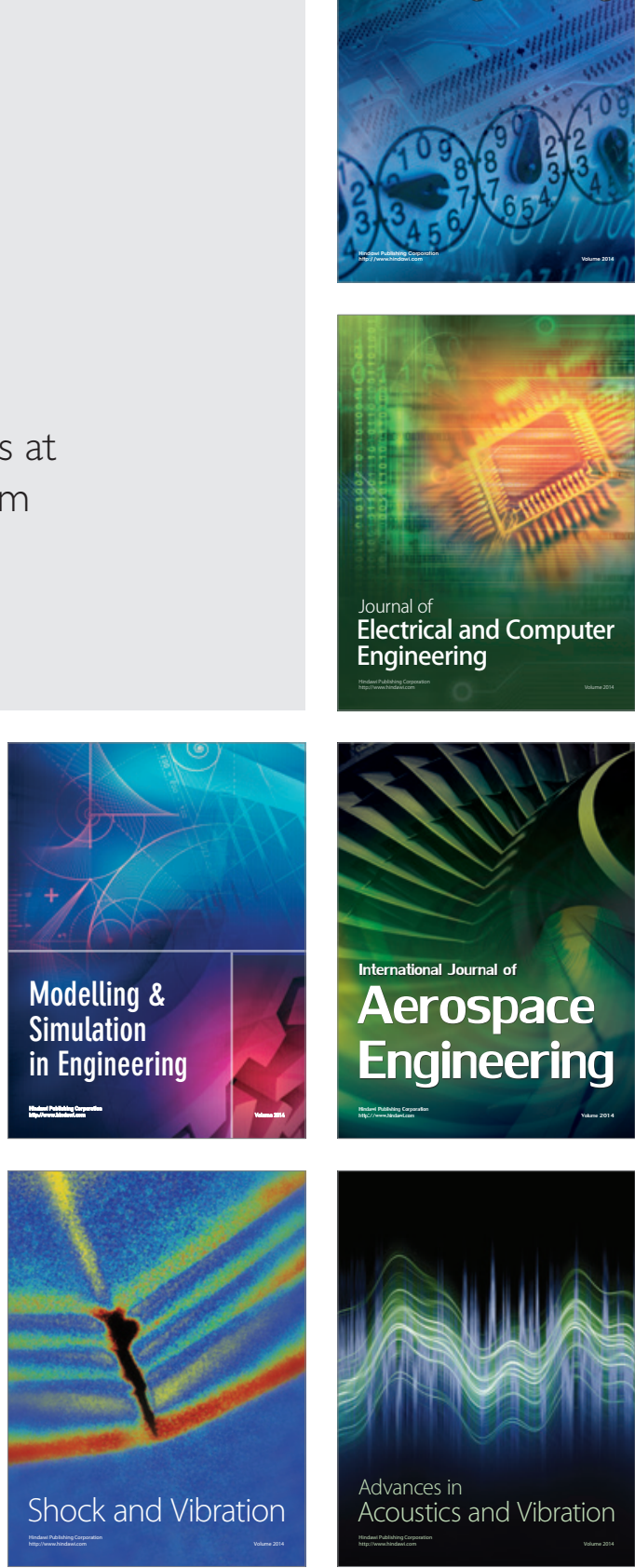